\title{
Temporal Dissociation between Hand Shaping and Grip Force Scaling in the Anterior Intraparietal Area
}

\author{
Marco Davare, ${ }^{1}$ Michael Andres, ${ }^{1,3}$ Emeline Clerget, ${ }^{1}$ Jean-Louis Thonnard, ${ }^{2}$ and Etienne Olivier ${ }^{1}$ \\ ${ }^{1}$ Laboratory of Neurophysiology and ${ }^{2}$ Rehabilitation and Physical Medicine Unit, Université Catholique de Louvain, 1200 Brussels, Belgium, and ${ }^{3}$ Unité de \\ Neurosciences Cognitives, Université Catholique de Louvain, 1348 Louvain-la-Neuve, Belgium
}

In humans, both clinical and functional imaging studies have evidenced the critical role played by the posterior parietal cortex, and particularly by the anterior intraparietal area (AIP), in skilled hand movements. However, the exact contribution of AIP to precision grasping remains debated. Here we used transcranial magnetic stimulation (TMS) to induce virtual lesions of the left and/or right AIP in subjects performing a grip-lift task with either hand.

We found that, during movement preparation, a virtual lesion of AIP had distinct consequences on precision grasping of either hand depending on its time of occurrence: TMS applied 270-220 ms before the fingers contacted the manipulandum altered specifically the hand shaping, whereas lesions induced $170-120 \mathrm{~ms}$ before contact time only affected the grip force scaling. The lateralization of these two processes in AIP is also strikingly different: whereas a bilateral lesion of AIP was necessary to impair hand shaping, only a unilateral lesion of the left AIP altered the grip force scaling in either hand.

The present study shows that, during movement preparation, AIP is responsible for processing two distinct, temporally dissociated, precision grasping parameters, regardless of the hand in use. This indicates that the contribution of AIP to hand movements is "effectorindependent," a finding that may explain the invariance of grasping movements performed with either hand.

Key words: hand shaping; transcranial magnetic stimulation; grip force scaling; finger; effector-independent; dominance

\section{Introduction}

In primates, the effortless dexterity contrasts with the complexity of the operations underlying object manipulation. The first prerequisite to grasp an object is to shape the hand in a posture adapted to its intrinsic properties (size, shape, and orientation) to position the fingertips correctly on the object (Jeannerod, 1986a; Goodale et al., 1994; Jeannerod et al., 1995). Then, fingertip forces have to be applied perpendicularly on the object and scaled very precisely to prevent the object from slipping or to avoid damaging it (Johansson and Westling, 1984, 1988). The grip force scaling is performed anticipatively and relies on the representation, or internal model, of the object dynamics linking applied force to motion (Flanagan and Wing, 1997).

The visuomotor transformations underlying hand shaping are implemented in a parietofrontal network, including the posterior parietal cortex (PPC) and premotor areas (PM) (Jeannerod et al., 1995; Castiello, 2005). Indeed, in monkeys, transient inactivation of either anterior intraparietal area (AIP) (Gallese et al., 1994) or the ventral part of PM (PMv) (Fogassi et al., 2001) has

\footnotetext{
Received 0ct. 17, 2006; revised March 6, 2007; accepted March 8, 2007.

This work was supported by grants from the Fonds Spéciaux de Recherche of the Universite Catholique de Louvain, the Fonds de la Recherche Scientifique Médicale, and the Fondation Médicale Reine Elisabeth. M.A. is a Postdoctoral Researcher at the Fonds de la Recherche Scientifique-Fonds National de la Recherche Scientifique. E.C. is a Research Fellow at the Fonds pour la Formation à la Recherche dans I'Industrie et dans I'Agriculture. We are grateful to Prof. L. Fadiga and Dr. J. Duqué for their comments on a previous version of this manuscript.

Correspondence should be addressed to Etienne Olivier, Laboratory of Neurophysiology, Université Catholique de Louvain, Avenue Hippocrate 54, 1200 Brussels, Belgium. E-mail: olivier@nefy.ucl.ac.be.

D0I:10.1523/JNEUROSCI.0426-07.2007

Copyright $\odot 2007$ Society for Neuroscience $\quad$ 0270-6474/07/273974-07\$15.00/0
}

been shown to impair the hand posture adapted to the object to grasp. In humans, comparable deficits have been reported in patients with PPC lesions (Jeannerod, 1986b; Jeannerod et al., 1995; Binkofski et al., 1998), and, recently, we demonstrated that a lesion of PMv distinctively impairs the finger positioning on the object (Davare et al., 2006). In contrast, little is known about the brain structures involved in the fingertip force scaling, and no clinical study has ever reported a selective deficit in grip forces after a restricted cortical lesion (Hermsdorfer et al., 2003; Nowak et al., 2003). Functional magnetic resonance imaging (fMRI) studies have suggested that AIP (Ehrsson et al., 2000, 2001; Imamizu et al., 2004) and the supplementary motor area (KuhtzBuschbeck et al., 2001; Bursztyn et al., 2006) play a role in the grip force scaling, possibly by building and/or storing internal models of object dynamics. The cerebellum is also classically regarded as a good candidate for performing these operations (Blakemore et al., 2001; Nowak et al., 2002; Monzee et al., 2004; Bursztyn et al., 2006).

The goal of the present study was to investigate further the role of the human AIP in hand shaping and fingertip force scaling and to determine the time course of its contribution during movement preparation. To address these issues, we quantified the deficits in precision grasping consequent to transcranial magnetic stimulation (TMS)-induced virtual lesions of the left and/or right AIP in healthy subjects performing a standard grip-lift task with either hand.

\section{Materials and Methods}

Seventeen right-handed (Oldfield, 1971) volunteers (22-29 years old) participated in this study after providing an informed consent. None of 
A

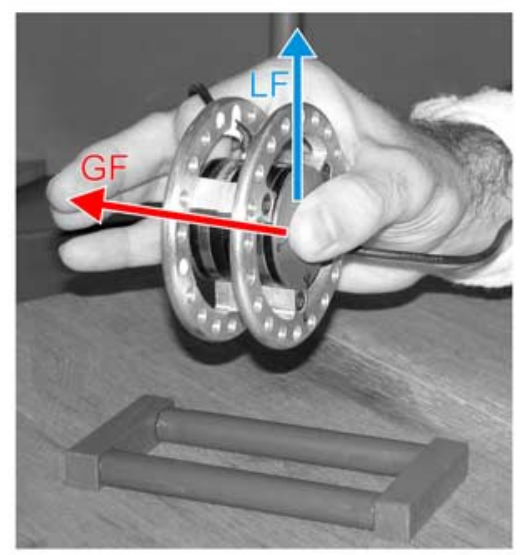

B

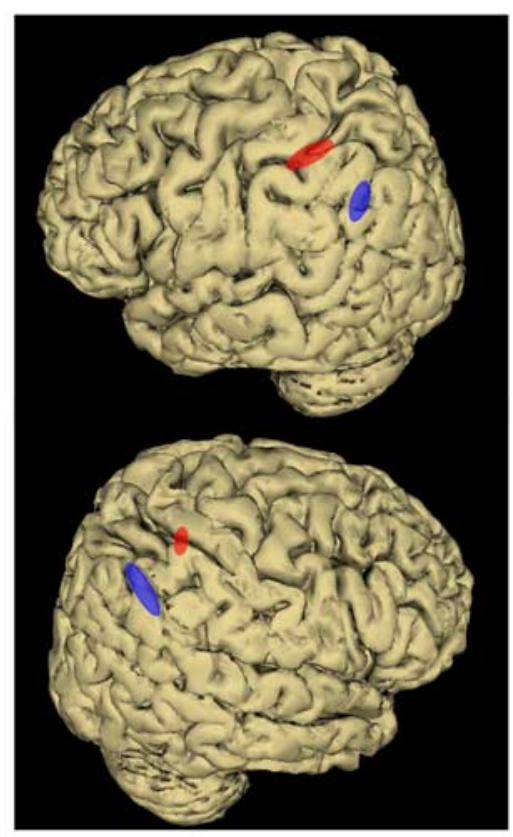

C
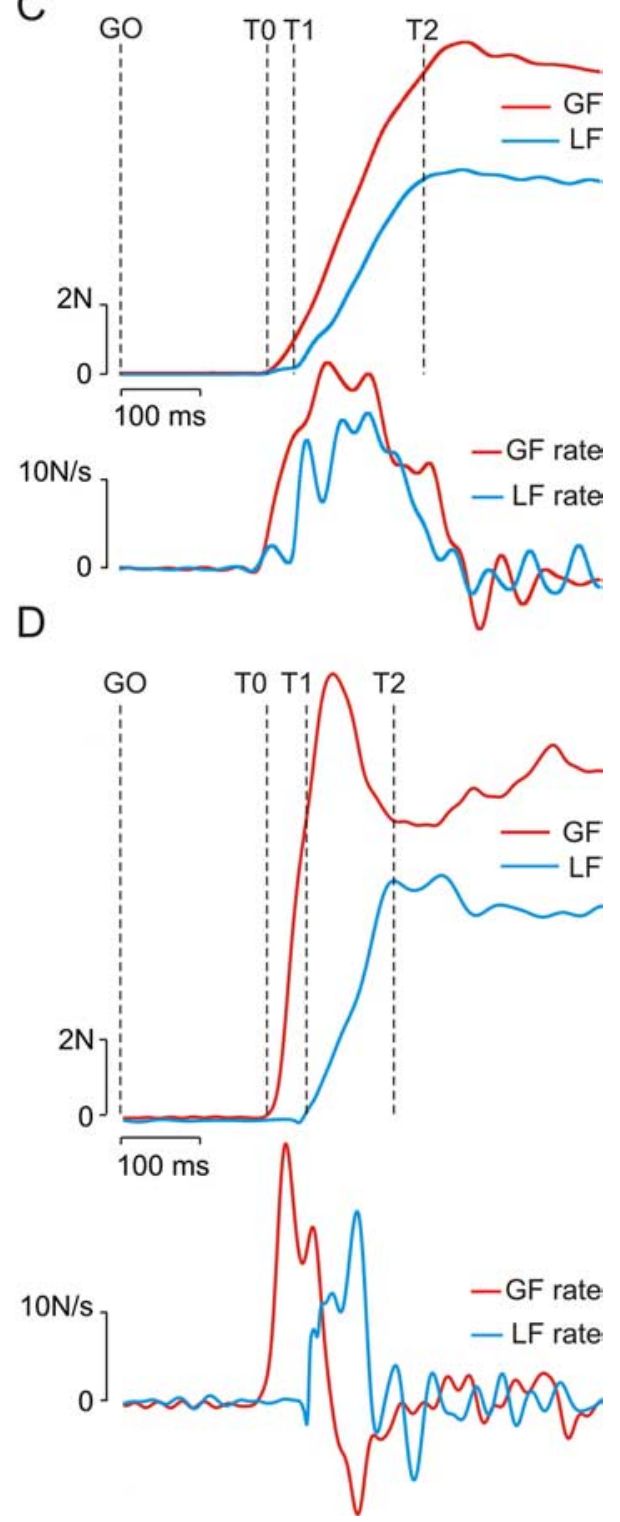

Figure 1. Experimental setup and fingertip force recordings. $\boldsymbol{A}$, Manipulandum used for the grip-lift task. Subjects had to lift this manipulandum between the thumb and index finger, by applying the minimum force required to prevent slipping. The manipulandum contained two three-dimensional force-torque sensors that allow to measure the $\mathrm{GF}, \mathrm{LF}$, and the fingertip positions. $\boldsymbol{B}$, Mean location of the stimulation points over AIP (red; $n=10$ ) and SMG (blue; $n=7$ ) in both the left and right hemispheres after a normalization into the MNI coordinate system. The ellipse center is located over the mean MNI coordinates of each stimulation site; the ellipse surface indicates the $95 \%$ confidence interval. C, Typical recording of fingertip forces in control conditions illustrating the grip-lift synergy. GF is in red, LF is in blue, and, shown below is their first derivatives (same color code). $\mathrm{T}_{0}-\mathrm{T}_{1}$, Preloading phase; $\mathrm{T}_{1}-\mathrm{T}_{2}$, loading phases. $D$, Effect of a virtual lesion of the left AIP induced with rTMS. Both GF and GF rate overshot control values and the preloading phase $\left(\mathrm{T}_{0}-\mathrm{T}_{1}\right)$ increased significantly after such a lesion. Same conventions as in $C$.

the subjects reported neurological impairments. They were all screened for adverse reactions to TMS by means of the TMS safety screen questionnaire (Keel et al., 2001). The experimental procedure was approved by the Ethics Committee of the Universite Catholique de Louvain.

Grip-lift task. The manipulandum weighted $575 \mathrm{~g}$ and consisted of two vertical parallel grip surfaces ( $40 \mathrm{~mm}$ diameter, $30 \mathrm{~mm}$ apart) (Fig. 1A), which covered three-dimensional force-torque sensors (Mini $40 \mathrm{~F} / \mathrm{T}$ transducers; ATI Industrial Automation, Garner, NC). Each sensor measured the three orthogonal forces $\left(F_{x}, F_{y}\right.$, and $\left.F_{z}\right)$ and torques $\left(T_{x}, T_{y}\right.$, and $T_{z}$ ). The force tangential to the grip surface [load force (LF)] was computed as the vectorial sum of $F_{x}$ and $F_{y}$. The force normal to the grip surface [grip force (GF)] was given by $F_{z}$.

The subject's hand laid relaxed on its ulnar edge, with the wrist in a mid-prosupinated position; both the thumb and index finger were positioned $\sim 4 \mathrm{~cm}$ apart from the manipulandum grip surfaces. Subjects were instructed to grip the manipulandum and to lift it by applying the minimum force required to avoid slips (Westling and Johansson, 1984). An auditory GO signal was delivered at the beginning of the trial and was followed, $\sim 3 \mathrm{~s}$ later, by another signal indicating the end of the trial.

Experimental procedure. Experiment 1 aimed at determining the contribution of AIP to precision grasping and its possible hemispheric dominance. Six subjects participated in this experiment that consisted of 12 blocks of 12 trials, preceded by a 24 trial training session without TMS. In six blocks, the grip-lift task was performed with the right hand and, in the other six blocks, with the left hand. For each hand, repetitive TMS (rTMS) was applied unilaterally either over the left (two blocks) or right (two blocks) AIP. In the two other blocks, rTMS was applied with the coil in a sham position, perpendicular to the scalp, over the left (one block) or right (one block) AIP.

Experiment 2 was designed to determine the time course of the left AIP contribution to the grip force scaling. Six subjects (four of them having participated in experiment 1 ) had to perform the same grip-lift task but only with the right hand, whereas a paired-pulse TMS was delivered at five different delays over the left AIP. This session consisted of 10 blocks of 15 trials. In five blocks, paired-pulse TMS was applied over the left AIP. In the five other blocks, TMS was delivered with the coil in a sham position

The goal of experiment 3 was to test for the selectivity of AIP virtual lesion to elicit deficits in precision grasping. To do so, rTMS was applied over either the left or right supramarginal gyrus (SMG), a PPC area that has been repeatedly found activated during tool use (JohnsonFrey et al., 2005; Culham and Valyear, 2006). Seven new subjects participated in this experiment; they had to perform the same grip-lift task with the right hand. This experiment consisted of six blocks of 12 trials. In four blocks, rTMS was applied over either the left or right SMG; in the two other blocks, sham rTMS was applied.

Experiment 4 (six subjects, four of them having participated in experiment 1) was designed to differentiate between the deficits in precision grasping resulting from a unilateral AIP lesion with those consequent to bilateral lesions. This experiment consisted of eight blocks of 25 griplift trials performed with the right hand. In half of the blocks, single TMS pulses were applied simultaneously over the left and right AIP (see below). In the other half, single TMS pulses were delivered with the two coils in a sham position.

In all experiments, the block order was pseudorandomly distributed among subjects.

Transcranial magnetic stimulation. In experiments 1 and 3, we used rTMS $(10 \mathrm{~Hz}, 500 \mathrm{~ms})$ triggered on the GO signal and applied, respectively, over the left or right AIP and over the left or right SMG. The rTMS trains were delivered through a figure-of-eight coil connected to a Rapid Magstim model 200 stimulator (Magstim Company, Whitland, UK). The coil was held tangential to the skull along a parasagittal line with the 
handle pointing backward. In these two experiments, the TMS intensity was set at $120 \%$ of the resting motor threshold (rMT). The rMT was defined as the minimum intensity that induced motor evoked potentials (MEPs) $\geq 50 \mu \mathrm{V}$ peak-to-peak in the first dorsal interosseus (1DI) in 5 of 10 trials; rMT was determined by using a single-pulse TMS delivered with the Rapid Magstim stimulator. The rMT were measured separately for the right and left 1DI; they were not statistically different $(t=0.78 ; p>$ 0.05). rTMS trains were separated by at least $12 \mathrm{~s}$.

In experiment 2 , a pair of TMS pulses, separated by $5 \mathrm{~ms}$, was delivered through a single coil by using a bistim module (Magstim Company). This paired-pulse stimulation was applied over the left AIP at five different delays after the GO signal, namely 50, 100, 150, 200, or 250 ms. The TMS intensity was set at $120 \%$ of the rMT determined for the right 1 DI.

Because the goal of experiment 4 was to produce bilateral lesions of AIP, we used two figure-of-eight coils connected to two single-pulse Magstim stimulators (Magstim Company); the two stimulators were triggered simultaneously. TMS intensity was set at 140\% of the rMT for the right 1DI because a pilot experiment had showed that, at this intensity, a single-pulse TMS applied unilaterally over the left AIP produced the same deficits in grip force as a $120 \%$ rTMS and a $120 \%$ paired-pulse TMS. Bilateral TMS was applied over the left and right AIP at five different delays after the GO signal, namely $0,50,100,150$, or $200 \mathrm{~ms}$. This approach also allowed us to investigate the time course of AIP contribution to precision grasping. Through the experiment, we constantly checked that the $140 \%$ TMS stimulation over the PPC did not elicit MEPs in the 1DI.

Stimulation sites. The coil was precisely positioned by means of a coregistration technique of the stimulation sites onto individual anatomical magnetic resonance images; this technique has been described in detail previously (Noirhomme et al., 2004; Davare et al., 2006; Zosso et al., 2006). This procedure was repeated for every subject before each experimental session. The individual coordinates of each stimulation site were normalized a posteriori into the Montreal Neurological Institute (MNI) coordinate system and averaged to compare the location of our stimulation sites with that of activation foci reported in fMRI studies.

To target AIP, the coil was positioned at the intersection between the intraparietal sulcus and postcentral sulcus. Indeed, a recent fMRI study has shown that visually guided grasping movements selectively and invariably activated, in every subject, an area located at the junction of these two sulci (Frey et al., 2005). The normalization procedure into the MNI coordinate system further established that we targeted the "parietal grasping region" evidenced by many other fMRI studies. Indeed, in the present study, the mean normalized MNI coordinates of AIP stimulation sites were, respectively, $-43.4 \pm 7.4,-39.1 \pm 7.1$, and $46.0 \pm 4.4 \mathrm{~mm}(x$, $y, z$, mean $\pm \mathrm{SD} ; n=10)$ and $44.0 \pm 3.7,-40.5 \pm 2.6$, and $44.6 \pm 4.8 \mathrm{~mm}$ $(n=10)$ in the left and right hemispheres (Fig. $1 B)$. These coordinates overlapped the location of AIP reported by fMRI studies in humans (Binkofski et al., 1999; Ehrsson et al., 2001; Frey et al., 2005; Begliomini et al., 2007) (for a comparison between the coordinates of AIP stimulation sites and those of activation foci found in fMRI studies, see supplemental Table A, available at www.jneurosci.org as supplemental material). Although this point is beyond the scope of the present study, it is likely that this parietal grasping region is the functional homolog in the human brain of the area AIP in monkeys (Grefkes et al., 2002; Grefkes and Fink, 2005).

In experiment 3, to target SMG, the coil was positioned just below the intraparietal sulcus, in front of the angular gyrus, a region that corresponds to the superior and caudal part of SMG. The mean normalized MNI coordinates of the SMG stimulation points were $-56.4 \pm 7.8$, $-59.7 \pm 3.9$, and $35.7 \pm 5.7 \mathrm{~mm}(n=7)$ and $56.2 \pm 8.8,-60.8 \pm 5.2$, and $39.2 \pm 8.5 \mathrm{~mm}(n=7)$ (Fig. $1 B)$ in the left and right hemispheres, respectively. Those stimulation sites overlapped consistently the activation loci found in SMG during skilled hand movements (Johnson-Frey et al., 2005; Culham and Valyear, 2006).

The Euclidian distance between the averaged coordinates of AIP $(n=$ $10)$ and SMG $(n=7)$ stimulation sites was $25 \mathrm{~mm}$.

Data acquisition and analysis. Force signals were digitized on-line at 1 $\mathrm{kHz}$ with a 12-bit 6071E analog-to-digital converter in a PXI chassis (National Instruments, Austin, TX). These signals were then low-pass filtered $(15 \mathrm{~Hz})$ with a fourth-order, zero phase-lag Butterworth filter. The GF and LF onsets were defined as the points at which the force value exceeded the mean $+2 \mathrm{SD}$ of the premovement value (Davare et al., 2006). The following temporal parameters were measured (Fig. 1C): (1) the reaction time, which is the time interval between the GO signal and the contact time of fingers with the manipulandum, defined as the GF onset, (2) the preloading phase duration $\left(\mathrm{T}_{0}-\mathrm{T}_{1}\right)$, which is the time interval between the GF and LF onsets, and (3) the loading phase duration $\left(\mathrm{T}_{1}-\mathrm{T}_{2}\right)$, which is the delay between the LF onset and the moment it equals the object weight. The following parameters were also computed: (1) the peak magnitude of the first derivative of GF (dGF/dt) and LF (dLF/dt), (2) the maximum coefficient of correlation between $\mathrm{dGF} / \mathrm{dt}$ and $\mathrm{dLF} / \mathrm{dt}$, and (3) the time shift that gave the maximum crosscorrelation. These two values were gathered for each individual trial by computing a cross-correlation function between $\mathrm{dGF} / \mathrm{dt}$ and $\mathrm{dLF} / \mathrm{dt}$ and were used to estimate the overall grip-lift synergy (Duque et al., 2003). The maximum coefficient of correlation permits to quantify the similitude between GF and LF, and the time shift provides a measure of the asynchrony between GF and LF, a positive value indicating that GF leads LF. Finally, the hand shaping was estimated by measuring the fingertip positions on the object, as by Goodale et al. (1994). To do so, we calculated the positions of the center of pressure (CP) of both the thumb and index fingertips on the manipulandum; the distribution of the finger positions in a given experimental condition was assessed by measuring the area of the ellipsoid in which $95 \%$ of the CPs were found (Davare et al., 2006).

Statistical analysis. Because repeated-measure ANOVA (ANOVA $A_{\mathrm{RM}}$ ) performed within sham blocks failed to show difference between blocks (all $F<1$ ), all data gathered in sham blocks were averaged for each experiment and used as controls. In experiment 1 , the effects of rTMS on temporal, dynamic parameters and fingertip positions and the hemispheric lateralization of the AIP contribution were analyzed by a three-

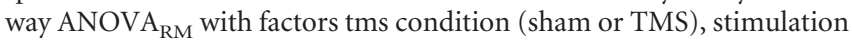
site (left or right AIP), and hand (left or right). In experiment 2, the time course of the TMS effects was analyzed by means of a two-way ANO$\mathrm{VA}_{\mathrm{RM}}$ with tms condition (sham or TMS) and stimulation delays (50, $100,150,200$, or $250 \mathrm{~ms}$ ) as factors. In experiment 3 , the effects of rTMS applied over SMG were analyzed by a two-way ANOVA $A_{R M}$ with factors tms condition (sham or TMS) and stimulation site (left or right SMG). Finally, in experiment 4, the effects of bilateral single-pulse TMS were analyzed by a two-way ANOVA $\mathrm{RM}_{\mathrm{RM}}$ with factors tms condition (bilateral sham or bilateral TMS) and stimulation delays (0,50, 100, 150, or 200 $\mathrm{ms})$. Tukey's tests were used for post hoc analysis.

\section{Results}

\section{Left AIP contribution to grip force scaling}

Experiment 1 showed that unilateral virtual lesions of the left AIP selectively impaired the grip force scaling in either hand. Indeed, rTMS applied over the left AIP led to an overshoot of both the grip force and its first derivative $\left(\mathrm{ANOVA}_{\mathrm{RM}}\right.$, tms $\times$ site interaction, both $F>4.32$, both $p<0.012$; Tukey's test, all $t>3.65$, all $p<0.035$ ) (Figs. 1C,D, $2 A$, Table 1), whereas rTMS applied over the right AIP had no effect (Tukey's test, all $t<1.85$, all $p>0.24$ ). In addition, we found that left AIP lesions delayed the onset of the load force (Figs. $1 D, 2 B$ ) as evidenced by a significant increase in both the preloading phase duration and time shift $(\mathrm{tms} \times$ site interaction, both $F>5.65$, both $p<0.008$; Tukey's test, all $t>$ 4.32, all $p<0.015$ ), whereas, again, right AIP lesions had no effect on these parameters (Tukey's test, all $t<1.05$, all $p>0.15$ ). Interestingly, the deficits in the grip force scaling and preloading phase duration after left AIP lesions were observed regardless of the hand used to grasp the manipulandum (no hand main effect and no interaction between hand and tms or site, all $F<1$ ). It is noteworthy that the hand shaping, as estimated by measuring the fingertip positions on the manipulandum (see Materials and Methods), was unaffected by unilateral AIP lesions (tms $\times$ site, all $F<1$ ) (Table 1). 
A

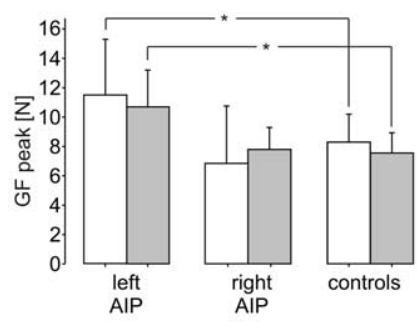

C

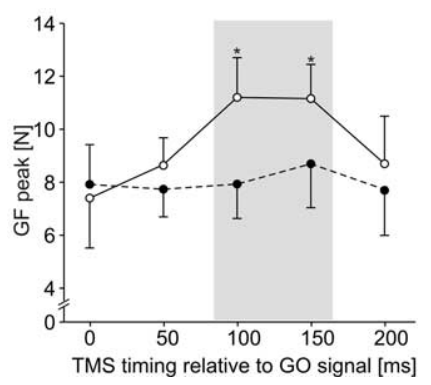

B

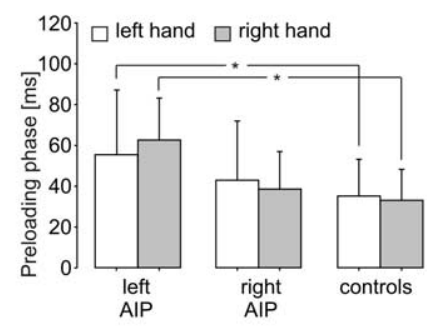

D

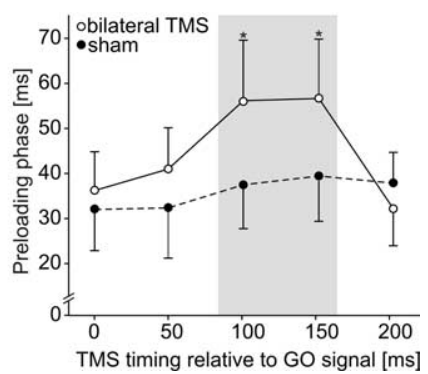

Figure 2. Effect of virtual lesions of the left AIP on the grip force scaling. $A$, Virtual lesions of the left AIP (experiment 1) yielded an increase in the grip force peak when compared with control conditions, regardless of the hand used to grasp the manipulandum. Lesioning the right AIP had no effect. $\boldsymbol{B}$, Effect of left AIP lesions on the preloading phase. A significant increase of the preloading phase duration was found after left, but not right, AIP lesions. Error bars represent one SD. ${ }^{*} p<0.05$. C, Time course of the AIP contribution to grip force scaling. Experiment 4 showed that the GF peak increased significantly only when TMS was delivered 100 and $150 \mathrm{~ms}$ (open circles) after the $\mathrm{G} 0$ signal when compared with control condition (sham; filled circles). $D$, Time course of the effects of AIP lesions on the preloading phase as determined in experiment 4. The effect on the preloading phase has the same time course as that found for the grip force peak. Same conventions as in $C$. Error bars represent one SD. ${ }^{*} p<0.05$.

Experiment 2 allowed us to confirm the results of experiment 1 and to determine the time course of the left AIP contribution to grip force scaling, because paired-pulse TMS were applied at five different delays (see Materials and Methods). Indeed, we found a significant tms $\times$ delays interaction (all $F>4.53$; all $p<0.015$ ), confirming that a unilateral lesion of the left AIP led to an increased grip force and grip force rate and to a longer preloading phase and time shift, but only when paired-pulse TMS was delivered 100 or $150 \mathrm{~ms}$ after the GO signal (Tukey's test, all $t>4.21$, all $p<0.019)$. Because in this task the RT was, on average, equal to $270.3 \pm 20.2 \mathrm{~ms}$ (mean $\pm \mathrm{SD} ; n=6$ ), these results indicate that

the AIP contribution to grip force scaling is critical $\sim 170-120 \mathrm{~ms}$ before the fingers contact the object.

\section{Contribution of AIP to hand shaping}

The absence of deficits in hand shaping after unilateral AIP lesions was unexpected and difficult to reconcile with results from monkey experiments showing that a unilateral inactivation of AIP altered the hand conformation (see Introduction). At least two hypotheses could be proposed to explain this discrepancy: (1) in humans, another PPC area is responsible for the visuomotor transformations underlying hand shaping; and (2) a unilateral lesion of AIP could be inadequate to evidence a deficit in hand shaping in humans.

To test this first hypothesis, we conducted experiment 3 in which rTMS was applied over either the left or right SMG, another PPC area known to be critically involved in skilled hand movements (Culham and Valyear, 2006). We found that virtual lesions of either the left or right SMG had no effect on the grip-lift task performed with the right hand $\left(\mathrm{ANOVA}_{\mathrm{RM}}\right.$, all $\left.F<1\right)$.

To address the second issue, we ran experiment 4 in which bilateral AIP lesions were induced by means of two single-pulse TMS applied simultaneously over both AIPs, at five different delays. We first confirmed the results of experiments 1 and 2 by showing that AIP lesions alter the grip force, grip force rate, and preloading phase duration when TMS was delivered 100 or 150 $\mathrm{ms}$ after the GO signal (tms $\times$ delays interaction, all $F>5.21$, all $p<0.025$; Tukey's test, all $t>3.89$, all $p<0.016$ ) (Fig. 2C,D, Table 2). Interestingly, we also found that, at other delays, bilateral AIP lesions affected the hand shaping, as evidenced by a larger dispersion of the fingertip positions on the manipulandum (Fig. $3 A, B$ ). Indeed, the area of the $\mathrm{CP}$ ellipses was found significantly larger for both the index and thumb only when bilateral TMS was delivered 0 and $50 \mathrm{~ms}$ after the GO signal (tms $\times$ delays interaction, both $F>4.65$, both $p<0.006$; Tukey's test, all $t>$ 6.32, all $p<0.032$ ) (Fig. 3C,D, Table 2). Because in this experiment the mean RT was $268.7 \pm 22.1 \mathrm{~ms}$ (mean $\pm \mathrm{SD} ; n=6$ ), these results indicate that the AIP involvement in hand shaping is critical $270-220 \mathrm{~ms}$ before contact time, i.e., $\sim 100 \mathrm{~ms}$ before it contributes to grip force scaling.

Although we did not test the consequences of a bilateral AIP lesion when the task was performed with the left hand, because neither a left nor a right unilateral lesion of AIP (experiment 1) altered the left hand shaping, the results of experiment 4 can be generalized to both hands.

Table 1. Effects of TMS on the parameters of grasping movements performed with either hand

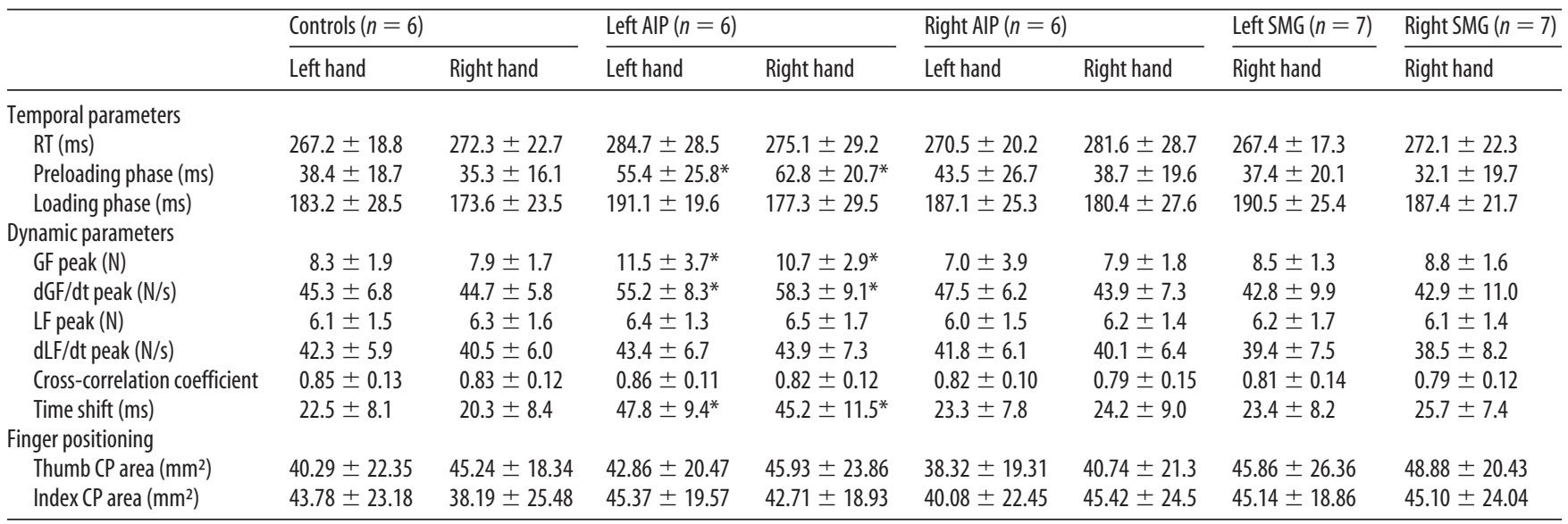

Mean \pm SD values of movement parameters gathered in the control condition of experiments 1 (sham), after rTMS over the left or right AIP (experiment 1) and after rTMS over the left or right SMG (experiment 3). RT, Reaction time; Thumb or Index CP area, ellipse in which $95 \%$ of the center of pressure of the thumb or index were found; $\mathrm{dGF} / \mathrm{dt}$ and $\mathrm{dLF} / \mathrm{dt}$, grip force and load force rates. ${ }^{*} p<0.05$, significantly different from control values. 
Table 2. Time course of the effects of AIP bilateral lesions on the precision grasping (right hand)

\begin{tabular}{|c|c|c|c|c|c|c|c|c|c|c|}
\hline & \multicolumn{10}{|l|}{ TMS delays } \\
\hline & \multicolumn{2}{|l|}{$0 \mathrm{~ms}$} & \multicolumn{2}{|l|}{$50 \mathrm{~ms}$} & \multicolumn{2}{|l|}{$100 \mathrm{~ms}$} & \multicolumn{2}{|l|}{$150 \mathrm{~ms}$} & \multicolumn{2}{|l|}{$200 \mathrm{~ms}$} \\
\hline & Sham & TMS & Sham & TMS & Sham & TMS & Sham & TMS & Sham & TMS \\
\hline \multicolumn{11}{|l|}{ Temporal parameters } \\
\hline $\mathrm{RT}$ (ms) & $273.4 \pm 22.1$ & $268.4 \pm 18.3$ & $258.7 \pm 21.4$ & $273.1 \pm 22.8$ & $274.1 \pm 25.9$ & $265.2 \pm 21.7$ & $270.5 \pm 20.2$ & $273.7 \pm 19.6$ & $278.4 \pm 21.4$ & $272.4 \pm 26.3$ \\
\hline Preloading phase (ms) & $32.2 \pm 8.2$ & $36.2 \pm 9.2$ & $32.5 \pm 11.2$ & $41.3 \pm 9.8$ & $36.1 \pm 7.6$ & $56.2 \pm 12.5^{*}$ & $37.8 \pm 7.7$ & $56.9 \pm 12.3^{*}$ & $36.5 \pm 6.5$ & $32.4 \pm 8.4$ \\
\hline Loading phase (ms) & $185.7 \pm 22.3$ & $177.3 \pm 18.4$ & $185.8 \pm 21.6$ & $190.2 \pm 26.1$ & $182.3 \pm 19.5$ & $175.4 \pm 21.5$ & $187.3 \pm 23.7$ & $172.4 \pm 19.3$ & $185.7 \pm 21.8$ & $192.4 \pm 18.7$ \\
\hline \multicolumn{11}{|l|}{ Dynamic parameters } \\
\hline GF peak (N) & $7.9 \pm 1.5$ & $7.5 \pm 0.8$ & $7.7 \pm 0.9$ & $8.5 \pm 0.7$ & $8.1 \pm 1.1$ & $11.2 \pm 1.3^{*}$ & $8.8 \pm 1.7$ & $11.0 \pm 1.2^{*}$ & $7.3 \pm 1.8$ & $8.6 \pm 1.8$ \\
\hline dGF/dt peak (N/s) & $44.6 \pm 6.1$ & $43.5 \pm 5.7$ & $45.4 \pm 5.8$ & $42.1 \pm 6.1$ & $44.8 \pm 6.0$ & $56.7 \pm 7.1^{*}$ & $45.8 \pm 6.2$ & $58.3 \pm 6.8^{*}$ & $47.4 \pm 5.7$ & $44.6 \pm 6.0$ \\
\hline LF peak (N) & $6.2 \pm 1.0$ & $6.4 \pm 1.2$ & $6.0 \pm 1.7$ & $6.2 \pm 1.2$ & $6.1 \pm 1.0$ & $6.3 \pm 1.4$ & $6.0 \pm 1.5$ & $6.0 \pm 1.3$ & $6.2 \pm 1.5$ & $6.3 \pm 1.6$ \\
\hline dLF/dt peak (N/s) & $38.3 \pm 5.7$ & $39.4 \pm 8.2$ & $39.2 \pm 7.4$ & $38.4 \pm 6.7$ & $39.7 \pm 7.3$ & $41.5 \pm 7.2$ & $37.9 \pm 6.0$ & $42.3 \pm 8.2$ & $38.5 \pm 6.9$ & $37.5 \pm 6.8$ \\
\hline Cross-correlation coefficient & $0.84 \pm 0.12$ & $0.82 \pm 0.10$ & $0.85 \pm 0.12$ & $0.87 \pm 0.13$ & $0.85 \pm 0.11$ & $0.83 \pm 0.15$ & $0.85 \pm 0.16$ & $0.87 \pm 0.12$ & $0.83 \pm 0.15$ & $0.80 \pm 0.14$ \\
\hline Time shift (ms) & $25.3 \pm 8.5$ & $27.3 \pm 10.3$ & $24.8 \pm 9.3$ & $28.3 \pm 10.5$ & $22.4 \pm 7.3$ & $44.7 \pm 10.3^{*}$ & $25.7 \pm 7.2$ & $47.2 \pm 9.7^{*}$ & $26.9 \pm 8.2$ & $29.3 \pm 9.4$ \\
\hline \multicolumn{11}{|l|}{ Finger positioning } \\
\hline Thumb (P area $\left(\mathrm{mm}^{2}\right)$ & $45.64 \pm 21.34$ & $66.37 \pm 25.76^{*}$ & $36.75 \pm 15.87$ & $94.57 \pm 39.21^{*}$ & $35.12 \pm 16.24$ & $39.85 \pm 17.24$ & $33.74 \pm 15.52$ & $37.65 \pm 15.21$ & $35.52 \pm 19.32$ & $38.93 \pm 16.8$ \\
\hline Index $\left(\mathrm{P}\right.$ area $\left(\mathrm{mm}^{2}\right)$ & $32.37 \pm 11.57$ & $76.98 \pm 20.35^{*}$ & $41.67 \pm 18.32$ & $82.65 \pm 37.12^{*}$ & $40.18 \pm 19.34$ & $36.54 \pm 13.87$ & $33.74 \pm 9.87$ & $39.52 \pm 12.28$ & $37.84 \pm 8.89$ & $38.21 \pm 12.51$ \\
\hline
\end{tabular}

Mean \pm SD values $(n=6)$ of movement parameters gathered in experiment 4 . RT, Reaction time; Thumb or Index CP area, ellipse in which $95 \%$ of the center of pressure of the thumb or index were found; $\mathrm{dGF} / \mathrm{dt}$ and dLF/dt, grip force and load force rates. ${ }^{*} p<0.05$, significantly different from control values.

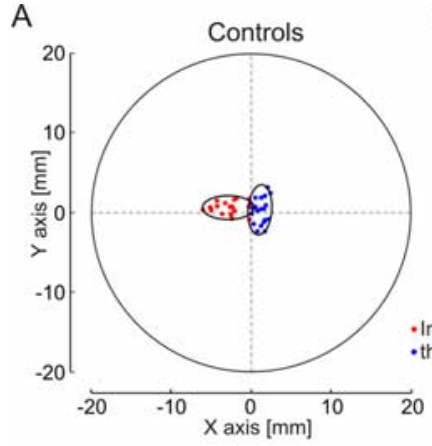

B Bilateral TMS over AIP

C

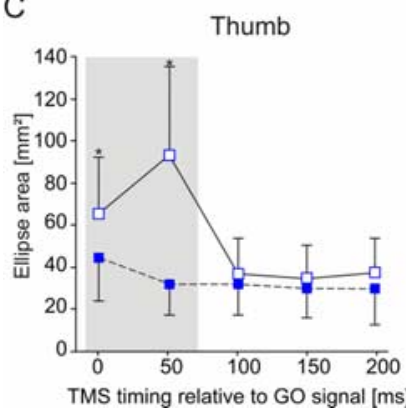

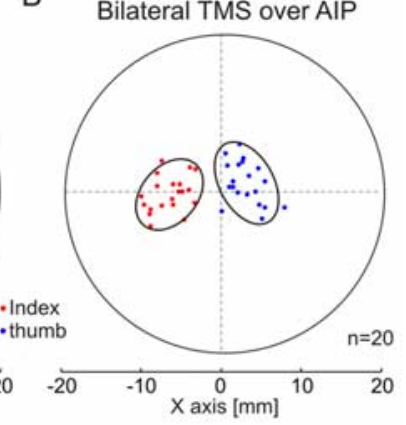

D

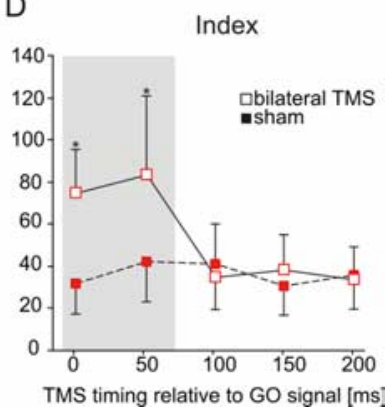

Figure 3. Effect of AIP virtual lesions on the hand shaping. $A$, Distribution of the index and thumb positions on the manipulandum for 20 control trials in one representative subject. The two graspable surfaces of the manipulandum were superimposed to represent the thumb (blue) and index (red) positions on the same graph. The ellipse center indicates the mean position of the fingertips, and the ellipse surface shows the area in which $95 \%$ of the fingertip positions were found. The subject's hand came from the right side of the figure. The mean index position was systematically more anterior than that of the thumb. $x$ - and $y$-axes represent, respectively, the distance from the manipulandum center along the horizontal and vertical axes. $\boldsymbol{B}$, Effect of a bilateral AIP lesion on finger positioning. A bilateral lesion of AIP led to a larger dispersion of fingertip positions on the manipulandum, suggesting that AIP contributes to hand shaping during grasping movements. Same conventions as in $A$. C, Time course of the contribution of AIP to hand shaping. The dispersion of the thumb positions was found increased only when TMS was applied, simultaneously over both AIPs, either 0 or 50 ms after the $\mathrm{G} 0$ signal (open squares); the filled squares indicate the control value (sham) gathered for each delay. $\mathbf{D}$, Time course of the effect of bilateral AIP lesions on the distribution of the index fingertip positions. Same conventions as in C. Error bars represent one SD. ${ }^{*} p<0.05$.

\section{Discussion}

The present study corroborates the view that, during precision grasping, the human AIP plays a key role in hand shaping; it also evidences for the first time the contribution of this area to the

fingertip force scaling. Additionally, we found that the involvement of AIP in controlling both the hand shaping and grip force occurs at different timings during movement preparation. We also demonstrated that the lateralization of these two processes is strikingly different: whereas only a lesion of the left AIP impaired the grip force scaling, a bilateral AIP lesion was necessary to perturb the hand shaping. Finally, we found that a unilateral SMG lesion had no effect on precision grip, in line with observations on apraxic patients showing that, although they have difficulties in using tools, their grasping movements may be preserved (Culham and Valyear, 2006; Daprati and Sirigu, 2006).

\section{AIP contribution to hand shaping}

In accordance with monkey experiments, we found that, during visually guided grasping, AIP plays a critical role in adjusting the hand posture to the object intrinsic properties (Gallese et al., 1994; Murata et al., 2000; Raos et al., 2006), supporting the view that AIP is involved in the visuomotor transformations underlying hand shaping (Jeannerod et al., 1995). However, in contrast to monkey experiments (Gallese et al., 1994), we found that a unilateral AIP virtual lesion failed to impair hand shaping. One possible explanation for this discrepancy is that TMS was unable to produce a substantial interference in AIP because of its location within the intraparietal sulcus. However, this appears unlikely because TMS delivered over the left AIP altered the grip force scaling; furthermore, we found that two single-pulse TMS applied simultaneously over both AIPs were sufficient to impair significantly the hand shaping, whereas a much stronger unilateral stimulation (rTMS, five pulses, $10 \mathrm{~Hz}$ ) failed to do so. These observations clearly suggest that, in humans, a bilateral lesion of AIP is necessary to produce a deficit in hand shaping, a finding consistent with previous TMS studies showing that a unilateral AIP lesion failed to alter the hand conformation (Glover et al., 2005; Tunik et al., 2005) except when the object size and orientation are unexpectedly changed. The findings that a bilateral lesion of AIP altered hand shaping, whereas a unilateral lesion was ineffective, indicate that each AIP can fully compensate for a lesion of the opposite one. This suggests that both AIPs are "interchangeable," regardless of the hand in use, although additional investigations will be needed to decipher the actual interactions between the two AIP in physiological conditions. Altogether, these observations support the view that the bilateral functional organization of AIP for hand shaping is a distinctive feature of the human PPC, as already suggested by fMRI studies showing a 
bilateral activation of AIP during unimanual precision grasping (Binkofski et al., 1999; Ehrsson et al., 2000, 2001; Culham et al., 2003; Castiello, 2005; Culham and Valyear, 2006).

However, the view that in humans the two AIP are interchangeable is somewhat inconsistent with results of clinical studies showing that, in some patients, a unilateral PPC lesion may impair hand shaping (Jeannerod, 1986b; Binkofski et al., 1998). One possible reason for this discrepancy is the difference between lesion size, much smaller in TMS studies (Nakamura et al., 2006). Another potential explanation for this discrepancy is that, whereas hand shaping deficits are usually quantified by measuring the maximal grip aperture during hand transport (Jeannerod, 1986b; Binkofski et al., 1998; Glover et al., 2005; Tunik et al., 2005), we used the final finger positions on the object, a more functionally relevant parameter (Goodale et al., 1994). It could therefore be hypothesized that the maximal grip aperture and finger positioning result from different visuomotor transformations, controlled independently by the nervous system.

Finally, we have evidenced that the AIP contribution to hand shaping occurs early during movement preparation, namely 0-50 ms after the GO signal, which, in the present study, corresponds to 270-220 ms before the fingers contact the object. Interestingly, in the same task, we recently found that PMv plays a key role in hand shaping $\sim 230-180$ before contact time, i.e., 50 ms after the AIP contribution (Davare et al., 2006); this supports the serial nature of the interactions between AIP and PMv during precision grasping (Jeannerod et al., 1995).

\section{Left AIP contribution to grip force scaling}

The present study also allowed us to identify, for the first time, a discrete cortical area involved in the grip force scaling. We found that virtual lesions of the left AIP led to an increase in grip force and grip force rate and a longer preloading phase; this replicated exactly the behavior of healthy subjects overestimating the weight of an object to grasp, for example, when it is replaced unexpectedly by a lighter one (Johansson and Westling, 1988). Because it is well known that the grip force scaling relies on the retrieval of an internal model of the object dynamics (Flanagan and Wing, 1997), the most likely explanation for our finding is that TMS has interfered somehow with the representation of graspable objects. However, the present study cannot decide whether the left AIP contains such an internal representation, recruits it, or processes sensory information that feeds it. So far, fMRI studies have also failed to distinguish between these different hypotheses: whereas Imamizu et al. (2004) have suggested that AIP may contain the internal models of object dynamics, Shadmehr and Holcomb have proposed that the role of AIP is to retrieve previously learned internal models, possibly stored in the cerebellum or in other cortical areas (Shadmehr and Holcomb, 1997; Bursztyn et al., 2006). Whatever the actual role of AIP, the present study shows that its contribution to grip force scaling is critical $170-$ $120 \mathrm{~ms}$ before the fingers contact the object, i.e., $\sim 100 \mathrm{~ms}$ after its involvement in hand shaping.

In contrast to the finding that the hand shaping can be processed by both AIPs, we found that only the left AIP is involved in the grip force scaling. This supports the hypothesis that dynamics may be a specialized function of the left hemisphere (Serrien et al., 2006). The left hemisphere dominance for skilled hand movements has been primarily demonstrated by clinical studies, in particular, for complex hand-tool interactions. Indeed, it is known that the left PPC critically contributes to hand-tool interactions (De Renzi et al., 1980; Koski et al., 2002; Culham and Valyear, 2006; Daprati and Sirigu, 2006), probably by retrieving the knowledge of object use (Koski et al., 2002). Importantly, the present study demonstrates that the left hemispheric dominance for skilled hand movements is not limited to high-level processes but also applies to elementary movement aspects, such as object dynamics representation.

\section{Conclusions}

The present study suggests that the AIP contribution to precision grasping is "effector-independent," i.e., independent of the hand used to perform the movement. Indeed, we found that each AIP can perform, for either hand, the visuomotor transformations of the object properties into a specific posture and that the left AIP is involved in scaling the grip force regardless of the hand used. Such an effector-independent organization supports the existence of a common cortical representation of hand movements, which has already been inferred from previous studies showing that grasping movements performed with the left or right hand are invariant (Tresilian and Stelmach, 1997; Grosskopf and Kuhtz-Buschbeck, 2006). This invariance has been demonstrated for both the kinematics (Grosskopf and Kuhtz-Buschbeck, 2006) and dynamics (Gordon et al., 1994) of grasping movements and is further supported by the present results showing a lack of differences between the grip-lift movements when performed with the left or right hand (see Table 1, control movements of experiment 1). However, the reason why, in the case of hand shaping, this "common cortical representation" is duplicated in the right and left AIP remains puzzling.

Finally, the finding that a single cortical area may process different movement parameters at separate timings questions the interpretation of fMRI studies when attempting to allocate a distinct process to a given cortical region. The present results definitely call for additional investigations of precision grasping with higher temporal resolution imaging techniques.

\section{References}

Begliomini C, Wall MB, Smith AT, Castiello U (2007) Differential cortical activity for precision and whole-hand visually guided grasping in humans. Eur J Neurosci 25:1245-1252.

Binkofski F, Dohle C, Posse S, Stephan KM, Hefter H, Seitz RJ, Freund HJ (1998) Human anterior intraparietal area subserves prehension: a combined lesion and functional MRI activation study. Neurology 50:1253-1259.

Binkofski F, Buccino G, Posse S, Seitz RJ, Rizzolatti G, Freund H (1999) A fronto-parietal circuit for object manipulation in man: evidence from an fMRI-study. Eur J Neurosci 11:3276-3286.

Blakemore SJ, Frith CD, Wolpert DM (2001) The cerebellum is involved in predicting the sensory consequences of action. NeuroReport 12:1879-1884.

Bursztyn LL, Ganesh G, Imamizu H, Kawato M, Flanagan JR (2006) Neural correlates of internal-model loading. Curr Biol 16:2440-2445.

Castiello U (2005) The neuroscience of grasping. Nat Rev Neurosci 6:726-736.

Culham JC, Valyear KF (2006) Human parietal cortex in action. Curr Opin Neurobiol 16:205-212.

Culham JC, Danckert SL, DeSouza JF, Gati JS, Menon RS, Goodale MA (2003) Visually guided grasping produces fMRI activation in dorsal but not ventral stream brain areas. Exp Brain Res 153:180-189.

Daprati E, Sirigu A (2006) How we interact with objects: learning from brain lesions. Trends Cogn Sci 10:265-270.

Davare M, Andres M, Cosnard G, Thonnard JL, Olivier E (2006) Dissociating the role of ventral and dorsal premotor cortex in precision grasping. J Neurosci 26:2260-2268.

De Renzi E, Motti F, Nichelli P (1980) Imitating gestures. A quantitative approach to ideomotor apraxia. Arch Neurol 37:6-10.

Duque J, Thonnard JL, Vandermeeren Y, Sebire G, Cosnard G, Olivier E (2003) Correlation between impaired dexterity and corticospinal tract dysgenesis in congenital hemiplegia. Brain 126:732-747. 
Ehrsson HH, Fagergren A, Jonsson T, Westling G, Johansson RS, Forssberg H (2000) Cortical activity in precision- versus power-grip tasks: an fMRI study. J Neurophysiol 83:528-536.

Ehrsson HH, Fagergren E, Forssberg H (2001) Differential fronto-parietal activation depending on force used in a precision grip task: an fMRI study. J Neurophysiol 85:2613-2623.

Flanagan JR, Wing AM (1997) The role of internal models in motion planning and control: evidence from grip force adjustments during movements of hand-held loads. J Neurosci 17:1519-1528.

Fogassi L, Gallese V, Buccino G, Craighero L, Fadiga L, Rizzolatti G (2001) Cortical mechanism for the visual guidance of hand grasping movements in the monkey: a reversible inactivation study. Brain 124:571-586.

Frey SH, Vinton D, Norlund R, Grafton ST (2005) Cortical topography of human anterior intraparietal cortex active during visually guided grasping. Brain Res Cogn Brain Res 23:397-405.

Gallese V, Murata A, Kaseda M, Niki N, Sakata H (1994) Deficit of hand preshaping after muscimol injection in monkey parietal cortex. NeuroReport 5:1525-1529.

Glover S, Miall RC, Rushworth MF (2005) Parietal rTMS disrupts the initiation but not the execution of on-line adjustments to a perturbation of object size. J Cogn Neurosci 17:124-136.

Goodale MA, Meenan JP, Bulthoff HH, Nicolle DA, Murphy KJ, Racicot CI (1994) Separate neural pathways for the visual analysis of object shape in perception and prehension. Curr Biol 4:604-610.

Gordon AM, Forssberg H, Iwasaki N (1994) Formation and lateralization of internal representations underlying motor commands during precision grip. Neuropsychologia 32:555-568.

Grefkes C, Fink GR (2005) The functional organization of the intraparietal sulcus in humans and monkeys. J Anat 207:3-17.

Grefkes C, Weiss PH, Zilles K, Fink GR (2002) Crossmodal processing of object features in human anterior intraparietal cortex: an fMRI study implies equivalencies between humans and monkeys. Neuron 35:173-184.

Grosskopf A, Kuhtz-Buschbeck JP (2006) Grasping with the left and right hand: a kinematic study. Exp Brain Res 168:230-240.

Hermsdorfer J, Hagl E, Nowak DA, Marquardt C (2003) Grip force control during object manipulation in cerebral stroke. Clin Neurophysiol 114:915-929.

Imamizu H, Kuroda T, Yoshioka T, Kawato M (2004) Functional magnetic resonance imaging examination of two modular architectures for switching multiple internal models. J Neurosci 24:1173-1181.

Jeannerod M (1986a) Mechanisms of visuomotor coordination: a study in normal and brain-damaged subjects. Neuropsychologia 24:41-78.

Jeannerod M (1986b) The formation of finger grip during prehension. A cortically mediated visuomotor pattern. Behav Brain Res 19:99-116.

Jeannerod M, Arbib MA, Rizzolatti G, Sakata H (1995) Grasping objects: the cortical mechanisms of visuomotor transformation. Trends Neurosci 18:314-320.

Johansson RS, Westling G (1984) Roles of glabrous skin receptors and sensorimotor memory in automatic control of precision grip when lifting rougher or more slippery objects. Exp Brain Res 56:550-564.

Johansson RS, Westling G (1988) Coordinated isometric muscle commands adequately and erroneously programmed for the weight during lifting task with precision grip. Exp Brain Res 71:59-71.

Johnson-Frey SH, Newman-Norlund R, Grafton ST (2005) A distributed left hemisphere network active during planning of everyday tool use skills. Cereb Cortex 15:681-695.

Keel JC, Smith MJ, Wassermann EM (2001) A safety screening questionnaire for transcranial magnetic stimulation. Clin Neurophysiol 112:720.

Koski L, Iacoboni M, Mazziotta JC (2002) Deconstructing apraxia: understanding disorders of intentional movement after stroke. Curr Opin Neurol 15:71-77.

Kuhtz-Buschbeck JP, Ehrsson HH, Forssberg H (2001) Human brain activity in the control of fine static precision grip forces: an fMRI study. Eur J Neurosci 14:382-390.

Monzee J, Drew T, Smith AM (2004) Effects of muscimol inactivation of the cerebellar nuclei on precision grip. J Neurophysiol 91:1240-1249.

Murata A, Gallese V, Luppino G, Kaseda M, Sakata H (2000) Selectivity for the shape, size, and orientation of objects for grasping in neurons of monkey parietal area AIP. J Neurophysiol 83:2580-2601.

Nakamura K, Hara N, Kouider S, Takayama Y, Hanajima R, Sakai K, Ugawa Y (2006) Task-guided selection of the dual neural pathways for reading. Neuron 52:557-564.

Noirhomme Q, Ferrant M, Vandermeeren Y, Olivier E, Macq B, Cuisenaire O (2004) Registration and real-time visualization of transcranial magnetic stimulation with 3-D MR images. IEEE Trans Biomed Eng 51:1994-2005.

Nowak DA, Hermsdorfer J, Marquardt C, Fuchs HH (2002) Grip and load force coupling during discrete vertical arm movements with a grasped object in cerebellar atrophy. Exp Brain Res 145:28-39.

Nowak DA, Hermsdorfer J, Topka H (2003) Deficits of predictive grip force control during object manipulation in acute stroke. J Neurol $250: 850-860$

Oldfield RC (1971) The assessment and analysis of handedness: the Edinburgh inventory. Neuropsychologia 9:97-113.

Raos V, Umilta MA, Murata A, Fogassi L, Gallese V (2006) Functional properties of grasping-related neurons in the ventral premotor area F5 of the macaque monkey. J Neurophysiol 95:709-729.

Serrien DJ, Ivry RB, Swinnen SP (2006) Dynamics of hemispheric specialization and integration in the context of motor control. Nat Rev Neurosci 7:160-166.

Shadmehr R, Holcomb HH (1997) Neural correlates of motor memory consolidation. Science 277:821-825.

Tresilian JR, Stelmach GE (1997) Common organization for unimanual and bimanual reach-to-grasp tasks. Exp Brain Res 115:283-299.

Tunik E, Frey SH, Grafton ST (2005) Virtual lesions of the anterior intraparietal area disrupt goal-dependent on-line adjustments of grasp. Nat Neurosci 8:505-511.

Westling G, Johansson RS (1984) Factors influencing the force control during precision grip. Exp Brain Res 53:277-284

Zosso D, Noirhomme Q, Davare M, Macq B, Olivier E, Thiran JP, De Craene M (2006) Normalization of transcranial magnetic stimulation points by means of atlas registration. Presented at the 14th Annual European Signal Processing Conference, Florence, Italy, September. 